 \\ International Journal of Otorhinolaryngology
}

\section{The Direction of Pediatric Sedation: A Contemporary and Historical Look at its Science, Art, Strengths and Shortcomings}

\begin{abstract}
This ma nusc ript takesa critic al look at the contemporary state and direction of sedation forthe pediatric dental patient from the viewpoint of an academician, researcher, and clinician with over thirty five years experience. When non-phamacological strategies prove ina dequate or inappropriate for managing the fearful and disuptive responses of the pediatric dental patient, conscious sedation is often selected as a viable alternative to unconscious techniques. The efficacy, safety, and predictability of this modality are described from both an historical and contemporary evidenced-based perspective. Despite the development of guidelines by multiple health provider disciplines, mishaps involving morbidity and mortality are reported which impact on institutional training and the direction for future use. Changes in philosophy, utilization, and the range of agents and dosing selected for overcoming childhood dental anxiety over the course of the past three decades are described. Emphasis will be given to the variability in training, experience, and current thinking within the educational arena and private practice setting. Lastly, discussion will include a review of cases resulting in fata lities, their etiology, and implic ations on drug availability, dosing recommendations, and projected future use of sedative modalities for children.
\end{abstract}

\section{Introduction}

The safe and successful use of sedative techniques for the provision of extensive treatment for many children can mean the difference between securing care at affordable rates versus the prohibitive costs for general anesthetics administered in a hospital or surgical center. This has potential to becoming financially devastating for those when insurance carriers deny medical benefits for the provision of dental treatment. Oral pediatric conscious sedation is considered a viable alternative pathway for management of minimal to moderate (and on limited occasions, extensive) treatment need for children lacking in cooperative ability when non-pharmacologic approaches prove inadequate or inappropriate.

Over the last three decades, few topics in pediatric dentistry generate greater diversity of opinion than the deployment of various forms of sedation to manage the behavioral manifestations of fear and disruptive behaviors of the pediatric patient. Because dentistry is openly viewed as unpleasant by most of society, and does involve a degree of discomfort, it is not surprising that dental treatment can represent a particularly frightening event for some children [1]. In contrast to many health care fields where second thought is rarely granted for any modality other than general anesthesia for completion of invasive and unpleasant procedures, expectations in dental practice, be it for adults or children, are that most procedures are conducted with the patient in a conscious state in an office setting. By definition, pre-cooperative children have immature cognitive skills, a restricted range of coping abilities, brief or negligible attention

\author{
John E. Nathan ${ }^{1,2 *}$ \\ ${ }^{1}$ Department of Otolaryngology/Dentistry, Northwestern University \\ Feinberg School of Medicine,Chicago, USA \\ ${ }^{2}$ Deptartment of Pediatric Dentistry, University of Alabama, \\ Birmingham, and Case Western Reserve University, Cleveland, USA \\ *Address for Correspondence \\ John E. Nathan, DDS, MDS., Associate Professor of Clinical \\ Otolaryngology,Department of Otolaryngology/Dentistry, Northwestern \\ University Feinberg School of Medicine,Chicago, 815 Commerce Drive, \\ Suite 220, Oak Brook, IL 60523, USA \\ Adjunct Professor, Deptartment of Pediatric Dentistry, University \\ of Alabama, Birmingham, and Case Western Reserve University, \\ Cleveland, USA, Tel: (630) 574-7336; Fax: (630) 574-9331; E-mail: \\ jnathandds@gmail.com \\ Submission: 15 December 2014 \\ Accepted: 16 January 2015 \\ Published: 20 January 2015
}

spans, and virtually no experience coping with stress. As result, they can be expected to be especially prone to maladaptive responses to anxiety provoking situations. For these children, conventional and traditional non-pharmacological behavior management strategies may often prove incapable of preventing or overcoming resistive and uncooperative behaviors. Selection of a pharmacologic approach is intended to limit or preclude the need for aversive measures and permit treatment in the least stressful manner possible for the child and dental team [2]. Use of any sedative technique is not without element of risk. Techniques which seek to overcome heightened levels of apprehension and patient resistance inherently carry potential to depress consciousness and adversely alter protective reflexes. Guided by science and an art which acknowledges the nuances and subtleties of individual patient differences, agents and dosage must be carefully chosen, and exercised with utmost diligence to insure patient safety.

This article has two objectives. First its intent is to serve as a practical reference and guide to facilitate practitioners' understanding of the responsibilities associated with the selection of a sedative modality as a non-mainstream technique mandating strict adherence to existing and ever-changing safety guidelines. To this end a synopsis of existing empirical and evidence-based support is included from a historical perspective to help the reader understand how far we have progressed in clarifying fundamentals of safety. While hundreds of sedation visits safely occur on a daily basis, incidents continue to appear (some with catastrophic outcomes) that reflect gross errors in clinical judgment and departures from standards of care. Second, with attention to the latter, its impact on contemporary teaching, on expectations for safe and effective use, definitions of what constitute clinical success, and comfort levels with respect to selection and use of appropriate agents and dosages is discussed. Instances of mortality are included for the purpose of acknowledging how future occurrences can be avoided and where continued study and prospective research is warranted. This article will be successful if it helps the reader focus on the long-term ramifications of the modalities we use for young patients as we best attempt to bridge the transition between precooperation and cooperative potential. 
Citation: Nathan JE. The Direction of Pediatric Sedation: A Contemporary and Historical Look at its Science, Art, Strengths and Shortcomings. Inter J Otorhinolaryngology. 2015;2(1): 8.

\section{The merit of oral pediatric conscious sedation}

The fact of the matter is that millions of people around the globe continue to avoid dental treatment for reasons related to discomfort, fear and situational anxiety it causes. Cost of care and its impact on access to care aside, there are adults and children who find it difficult if not impossible coping with the stress of dental treatment. The vast majority of the population has the capacity to accept and cope with treatment. For this segment, conventional communication and mainstream approaches are generally satisfactory.

For those lacking in cooperative potential, however, alternate strategies may become warranted. This may include pharmacological, conscious or unconscious techniques. While a preponderance of children possess cooperative potential and can be readily convinced to accept the rigors of dental treatment, there are some who by virtue of age, cognitive skills, or negative previous experience, resist every non-pharmacological approach offered. Even the most skilled dental provider may only safely render care to these individuals with adjunctive therapeutic means [2,3]. General anesthetics, while highly predictable and successful in permitting completion of treatment, are not without risk and occur at great cost. Optimally stated, oral conscious sedation for some children has potential to ameliorate apprehension, permit delivery of care to a minimally depressed conscious individual, in a setting where costs are significantly reduced. That said, there is no assumption that this modality escapes or excludes risk; mandated is that all efforts be directed at circumventing the limitations of the oral route of administration with strict adherence to existing guidelines for its safe use.

\section{The art, subtleties and nuances of pediatric sedation}

Not all issues can be determined or explained by scientific exploration. With respect to the "art" of pediatric sedation, it suffices to say that this implies a degree of intangible expertise based on extraordinary observational skills of a clinician and his or her experience to most accurately assess the needs of a given patient when selecting agents and dosages [4-8]. Selection of optimal therapeutic dosing of a given agent on the basis of the duration of action needed, the level of apprehension/resistance to be overcome, and the extensiveness/invasiveness of the procedure undertaken are key elements which separate the novice from the expert [4]. If one can stipulate that an optimal goal when utilizing sedative techniques is to maintain patient consciousness, (from pre-op through full recovery), obtund interfering movement, eliminate the need for restraint in a persistent manner, it begs the question of why approximately $60 \%$ of todays' pediatric specialists (Vargas et al. Wilson and Nathan) $[7,8]$ consider sedation still successful when persistent restraints are necessary. Those of this persuasion have low expectations for what constitutes success or fulfillment of optimal levels of sedation for their patients. It would seem reasonable that no parents in private practice would request to observe their child undergoing treatment, having been medicated, yet still require persistent application of physical restraint to complete treatment. From a pragmatic perspective, it might be understandable if a parent were to ask why they were assessed a sedation fee under circumstances where gross error in clinician judgment resulted in inadequate sedation.
While somewhat over simplified, the literature suggests this is more often the rule rather than exception. For a surprising number, a majority of pediatric dental specialists regard inadequate sedation as clinically successful if despite need for restraint, treatment is completed and use of the operating room is avoided. What constitutes an acceptable frequency for which optimal sedation can be expected to occur? $50 \%$ represents no better than a coin toss.70, 80 , or $90 \%$ ? Should pediatric specialists accept results which might be characterized as best as mediocre, or insist on more productive outcomes? From the perspective of a clinician with skills to best judge optimal agent and dosing, expectations for success, defined by minimal or no need for restraint to perform treatment, seems appropriate and reasonably achievable.

\section{The shortcomings of pediatric oral sedation}

Shortcomings of oral sedation for the pediatric patient fall under several venues. These include the oral route of administration, the limitations in its arsenal of pharmacologic agents, restricted in reversal capabilities, the limited availability of hard evidence of its efficacy and safety for varying levels of apprehension, and lastly wide variation in clinician training, exposure to its use, and proficiency/ comfort level in recognition and management of an adverse reaction.

\section{Oral route of administration}

While the virtues of parenteral (IV, IM) administration of drugs include opportunity for titration to the individual responses of a given patient, practicality for its usage is highly diminished for the pediatric patient. Prohibitive liability costs render use of parenteral routes in a pediatric office or clinic setting as non-feasible. The frequency, incidence, and severity of untoward reactions far exceed those from the oral route. While the oral route poses a generally simplistic ease of administration pending the palatability of its formulations and ingestion compliance of its subjects, its variable absorption, delay in onset often requiring prolonged latent periods, low potency and limited value of gastric lavage in the event of a problem, all pose inherent limitations for its use. Nevertheless, its use and popularity for the pediatric patient has enabled literally thousands of cases annually to avoid the need for general anesthesia, and/or application of physical restraint/aversive measures to permit dental treatment under conditions where non-pharmacologic approaches prove insufficient [3].

\section{Oral agents available for the pediatric agent}

While numerous agents of variable degrees of potency including sedatives/hypnotics, antihistamines, benzodiazepines, barbiturates, narcotics and dissociative anesthetics have been reported, rarely is substantive data from controlled study found to support efficacy and safety. As such discussion is restricted to those most frequently used and inclusions of agents lacking evidence are considered to fall outside the scope of this paper. Similarly, in-depth discussion of the pharmacodynamics of individual agents falls outside the scope of this paper. The ideal agent or combination has yet to be identified that is without downside. Prospective data to provide clarification of what constitutes predictable and safe dosing limits for varying levels of anxiety and resistance has yet to be presented. Exceptions include retrospective reports of multiple sedation experiences by a few researchers [9-11]. As a result, focus below will center on the use 
Citation: Nathan JE. The Direction of Pediatric Sedation: A Contemporary and Historical Look at its Science, Art, Strengths and Shortcomings. Inter J Otorhinolaryngology. 2015;2(1): 8.

of chloral hydrate and midazolam combinations, agents which have been most extensively studied.

\section{Clinician and training variability}

In addition to the above shortcomings, variability in clinician experience and training using a limited arsenal of agents, some being highly restricted in selection and dosing, no doubt contributes to a diverse array of sedation use in both academic and private practice settings. Surveys of pediatric dentists' use of sedation [7,8,10-24] have provided insight into backgrounds of both program directors and graduates on the basis of their training experiences using sedation protocols their institutions permit. For Program directors with a command of the sedation literature and vast experience using a diverse arsenal of agents, training experiences for residents can be expected to be optimal. For inexperienced program directors, reluctance to use either a diverse range of agents or dosing for varying levels of childhood anxiety and resistance, training experience of residents in sedation can be commensurately reduced. Based upon recent surveys, harsh institutional restrictions are reported which seriously limit therapeutic dosing to the extent that few if any successful sedation experiences were encountered. Clinicians reporting this end result from their training expressed they were not likely to make use of sedation in clinical practice and would likely opt for general anesthesia when encountering patient resistance. Indications from these clinicians showed preference to increased use of restraint or "protective immobilization" over sedative modalities. Alternatively, those with broad- based sedation training experiences indicated they anticipated making use of sedation to a greater extent [8].

Described below is potential impact of these shortcomings on guideline compliance and regulatory action to enhance safety as well as implications for enhancement of sedation teaching curriculum.

\section{Historical Use in Pediatric Dentistry and Evidence- Based Support}

To a large extent, prior to 1970 , there was a paucity of sources to provide substantive data to identify agents and sedative dosing for the pediatric patient. Most reports were anecdotal in origin; [25-29] occasionally text recommendations [26] were available to identify single agents or combinations intended to help reduce or preclude the application of aversive measures to treat uncooperative or resistive children. Rarely found are studies which examined the anxiety process and the pediatric dental patients' responses prospectively to regimens in any degree of controlled study.

Numerous factors account for the shortcomings of pediatric dental sedation research, applicable before 1970 and extending to the current day. Studies are difficult to design and conduct; patient selection criteria are deleted, or often at best vague. Design flaws often contain confounding variables (use of restraints, or confounding adjunctive agents such as nitrous oxide in fixed concentrations) compromising interpretation, adequate sample sizes are difficult to obtain, qualitative and quantitative assessment of behavioral and physiological patient responses are complex; other problematic issues find sequential and longitudinal study highly demanding and labor-intensive; further complicating is there is virtually no funding or priority given for this line of research. Despite these problematic issues, need for controlled study remains essential to advance our knowledge.
Several clinicians offered insights early in the sedation literature. Lampshire [30] reported significant improvement in the sedative experience by providing what he called "balanced sedation." It was his belief that all agents when used alone possessed certain benefits as well as disadvantages, that the addition of a second or third agent to offset the downsides of a given primary agent had potential to improve the safety and quality of sedations in children. This theoretical construct serves as a basis for today's combinations [4].

Following that logic, Robbins [37] reported the benefits of combining an anti-emetic agent with the sedative/hypnotic, chloral hydrate $(\mathrm{CH})$. He found that the addition of the anti-emetic significantly improved the quality of the sedation and permitted a reduction to half of the primary sedative, while minimizing the incidence of nausea. This finding has relevance today in that the addition of an anti-emetic and/or opiod has been the subject of numerous clinical trials in the last three decades to identify safe and more efficacious regimens.

Musselman and McClure [27,28] offered a practical approach to agent and dosage selection on the basis of apprehension levels/ resistance to be overcome and the duration of action required.

They described the need for "preventive medication" for those with milder levels of anxiety, and "management medication" necessitating higher dosages when confronting moderate and severe apprehension or resistance. To date, only a few clinical trials [4] acknowledge differences in patient apprehension levels and the need for dosage ranges for a given agent or combination.

In the late 1970's, alphaprodine (Nisentil R) was found to offer significant potential benefits in the field of obstetrics for its profound analgesic characteristics. It was adopted by many as an injectable agent with a potential capacity to rapidly obtund disruptive child behaviors in the dental setting.

Its use and misuse was later recognized as its accompanying respiratory depressant capabilities were the cause of morbidity and mortality. Recognition of these effects resulted in termination of its use and production. Goodson and Moore [29] reported 14 cases involving instances of catastrophic outcome and morbidity from the excessive use of this potent narcotic and local anesthesia, both presumed to be safe by pediatric dental practitioners. This landmark paper along with Moore described below put in motion the need to develop safety guidelines, define suitable and responsible levels of sedation, the need for thorough pre-treatment patient physical evaluation, patient monitoring requirements, and personnel and facility needs regarding preparedness for recognition and management of adverse reactions should any occur.

Moore [30] et al. (1984) compared 20, 40, and $60 \mathrm{mg} / \mathrm{kg}$ dosages of $\mathrm{CH}$ to a placebo with and without adjunctive nitrous oxide. An assessment of patients' ability to reactively maintain their airway with differing sedative dosages was included. Despite significant design flaws (no patient selection criteria defined, and fixed concentration of nitrous oxide used), which impaired interpretation of primary drug effects, the merit of this study was that it drew needed attention to patient response assessment and safety, not merely whether medication was effective. 
Citation: Nathan JE. The Direction of Pediatric Sedation: A Contemporary and Historical Look at its Science, Art, Strengths and Shortcomings. Inter J Otorhinolaryngology. 2015;2(1): 8.

\section{ISSN: 2380-0569}

The 1980's saw adoption of a research methodology [31-36] which gained momentum, if not simply concatenated authority, among several research locales. While no mention was made to clarify patient selection criteria in these trials, weak but nevertheless conscientious efforts were directed at assessment of patients behaviors,(crying , sleep, and movement) as determinants of sedation efficacy. All subjects received $50 \%$ concentrations of nitrous oxide, (regardless of need or titration) and were bound in papoose boards. Statistical and descriptive analyses of movement, (from inadequate sedation or agitation from being bound), incidence of nausea ( from excessive primary medication vs. excessive nitrous oxide), and calmness ( from optimal sedation vs. over- sedation), were at best, compromised. As such, the opportunity to draw valid conclusions regarding efficacy of the primary agent and its dosage schedule limited the value of these studies.

\section{Most commonly used and studied agents}

The most frequently utilized agent for sedation of children has been chloral hydrate for extensive treatment and lengthy visits. Anecdotal and manufacturer recommendations suggested the use of $25-50 \mathrm{mg} / \mathrm{kg}$. Trapp [26] (1982) suggested the oral dosage be extended from $50-70 \mathrm{mg} / \mathrm{kg}$ because of frequently experienced failure of $50 \mathrm{mg} /$ $\mathrm{kg}$ hypnotic dose to effectively sedate most apprehensive patients. Several studies [37-46] followed which explored the effectiveness of elevation (and reduction) in its dosage, most of which reported enhanced sedation, but not without increased incidence of emesis, prolonged somnolence, and airway concerns.

In an effort to reduce or eliminate these side effects, Nathan and West [9] hypothesized in a retrospective study, similar to Lampshire's premise, that the addition of a narcotic to the sedative-antiemetic combination could provide analgesia and improved sedation without induction of depressed consciousness and somnolence by permitting reduction rather than elevation in the sedative dosage. Hasty et al. [38] prospectively in one of the rare well-designed and controlled studies to date reported similar results. The regimen of $\mathrm{CH}$-hydroxyzinemeperidine has since been among the most studied and utilized until recent times [35,37-46].

Midazolam, more potent than diazepam, a short-acting benzodiazepine with capacity for reversal (unlike $\mathrm{CH}$ ), has gained popularity in anesthesiology as a premedication for children prior to a general anesthetic, and as a sedative agent to control child dental behavior. Despite considerable study, [47-51,60] it is considered at best unpredictable with respect to efficacy and duration of action. No consensus appears to exist among clinicians with respect to its dosing. While most report virtually no success with dosages under $0.7 \mathrm{mg} / \mathrm{kg}$, surveys have reported that several institutions today do not permit exceeding $0.5 \mathrm{mg} / \mathrm{kg}$.

The addition of anti-emetic as a potentiator has been studied as well as the addition of narcotic to prolong its duration and improve sedative effects. Despite some improvement in obtunding interfering behaviors, midazolam with or without narcotic and anti-emetic remains ill-construed as a combination for visits requiring anything but ultra short durations of action [60]. It is belief of many that no agent or combination exists that surpasses the range of safety and duration of action of chloral hydrate in combination with anti-emetic and meperidine.
In response, however, to misuses of $\mathrm{CH}$ over the past few years, manufacture of the oral form of $\mathrm{CH}$ has ceased and several states and institutions have banned its use in any form. The impact of which is that a void in the arsenal of agents available to provide lengthy visit (greater than 30-75 minute working time) sedation exists. As result, a significant increase in the utilization of general anesthesia is occurring. At present, $\mathrm{CH}$ remains available in tablet and powder form. Its continued use requires compounding by a trained pharmacist, able to create a pleasant tasting oral elixir formulation, often requiring less volume than its original manufacture's unit measure of $100 \mathrm{mg} / \mathrm{cc}$. Those pediatric dentists preferring to use this medication in combination with anti-emetic and narcotic make use of such pharmacists. Nathan reported using a range of $25-35 \mathrm{mg} / \mathrm{kg}$ $\mathrm{CH}$ and reporting success rates of $95 \%$ in over 3000 cases over the last 25 years (without induction of somnolence or need for transient or persistent use of physical restraints) for moderately apprehension and extensive treatment need $[3,52,53]$.

\section{The impact of disciplinary guidelines on the use of pediatric sedation in dentistry}

Prior to 1985, within and outside a pediatric dental context little attention was given to how patients, young or old, responded to various sedation regimens. Pre-treatment physical evaluation, preoperative and intra-operative monitoring of vital signs and protective reflexes were lacking. Pts were largely selected as being in need of sedation to avoid general anesthetics and upon administration of agents, were judged ready for treatment after achieving a somnolent state during a latent period. Reports of mishap, over-dosage, let alone morbidity and mortality had just begun to emerge.

During and subsequent to 1985, formal guidelines have been presented by various health care disciplines. These include the American Academy of Pediatric Dentistry, American Academy of Pediatrics, American Society of Anesthesiology, the American Dental Association, and the American Association of Oral and Maxillofacial Surgery. Some have occurred as collaborative efforts. In some cases, opinions and policy were offered independently without seeking input from related groups, with and without contextual evidence [54]. Regardless of discipline, these included recommendations for agent selection, physical evaluation and airway assessment, pre-, intra-, and post-operative patient monitoring, personnel and facility training and equipment, and emergency management protocols. Further elaboration of specifics is considered outside the realm of this paper.

No viable arguments exist against the refinement of safety guidelines to insure the safety of children. Each discipline shares in the responsibility to do all within its power and imagination to accomplish this end. Despite such, it is clear that numerous incidents continue to occur, albeit few, where providers continue to ignore safety and monitoring recommendations. Should this trend continue, it seems logical and appropriate for disciplines to continue efforts directed at tightening standards which inherently make the use of sedative techniques more challenging, impractical, if not impossible. With respect to the use of sedation in pediatric dentistry, trends report that its use has been in decline for the past two decades [54]. If such occurs where evidence finds competent implementation injurious, its use should move in that direction. If the discipline of pediatric dentistry chooses to lessen its quest to promote and conduct sound 
Citation: Nathan JE. The Direction of Pediatric Sedation: A Contemporary and Historical Look at its Science, Art, Strengths and Shortcomings. Inter J Otorhinolaryngology. 2015;2(1): 8.

research to enhance safety, and not take a more proactive role in cases involving abuse of its guidelines, further decline in the use of sedation can presumably be anticipated.

Despite the development of safety recommendations, which serve only as guidelines, acceptance and compliance remains today short of universal. As stated above, an axiom often claimed by disciplines inclusive of the American Academy of Pediatric Dentistry, and without claims to the contrary, is that when safety guidelines are followed, no adverse outcomes have been reported. The extent to which mishaps have continued to occur on a national basis since 1985, no doubt has played a role in recent and current teaching philosophies and constraints in the use of various agents or therapeutic dosages. Despite a lack of clinical data, the American Academy of Pediatrics and American Society of Anesthesiology have extended recommendations and restrictions beyond the monitoring requirements originally proposed in 1985 by the AAPD to the extent that such implementation further tightens control on the use of sedation. While not stated explicitly, such guidelines external to pediatric dentistry appear directed toward those who continue to ignore safety and monitoring recommendations [54] Questions have been raised if these changes will prevent adverse outcomes, or will they serve to drive more conscientious practitioners from sedation. Data seems to suggest lesser use of sedation in pediatric dentistry and greater use of general anesthesia. Is this the appropriate direction for pediatric sedation? Similarly, litigation resulting from such departures, has justifiably served as stimulus for increased state and institutional regulation, by virtue of their obligation to protect the public, as to what constitutes acceptable agents for the purpose of sedating children. Several states have, by virtue of the actions of a few, involving gross negligence, malpractice, and intentional departure from safety guidelines, eliminated the use of historically believed viable and safe agents.

\section{Contemporary impact on the teaching of sedation in advanced training programs}

Despite recent advancement from some of these trials, intended to enhance clinical success, continued growth in our knowledge, competency, and comfort range appears to be in a direction of stagnation, if not regression. Surveys $[8,19,21,23,24]$ indicate a growing propensity in training programs which seek to make use of lower potency agents and /or low-end drug dosing. Such actions are speculated to reduce risk of inadvertent induction of deeper planes of sedation, and are accompanied by an acceptance of the need for physical restraints and aversive measures on challenging children.

Wilson and Nathan (2011) [8] surveyed program directors and new graduates of postdoctoral training programs in pediatric dentistry to ascertain current teaching curricula, sedation experience, and comfort levels in the use of sedation. They point out the knowledge gained from numerous sedation studies to date contribute to a deficiency of a widely accepted body of clinical knowledge and applicability. Simple dose-response studies, a basic pharmacological tenet, on the individual effectiveness of individual medications or common combinations in children are rare. The extent of training of pediatric dentists and their experience in sedation varies widely. This ranges from virtually none to extensive. Those with little or no exposure raises questions of accreditation compliance under circumstances where experience using a variety of agents does not occur.

Conceptual definitions of what constitute clinical success vary widely. While some encounter experience with a diverse arsenal of agents and dosages for varying levels of apprehension and duration of action, others report exposure only to the use of a single agent with severe restrictions imposed on using moderate let alone upper limits of dosing. $47 \%$ of programs surveyed indicated using $\mathrm{CH}$ alone for their sedations. Use of this GI-upsetting agent without anti-emetic has long been known as inappropriate and unproductive. This finding was especially puzzling if not disconcerting. One explanation might be in the extent of sedation background and experience these training program directors have. Pervasive use of midazolam, with and without additional agents in dosages were reported [8] (many restricting dosage to no greater than $0.5 \mathrm{mg} / \mathrm{kg}$ while others permitting up to $1.0 \mathrm{mg} / \mathrm{kg}$ on routine basis). $94 \%$ of training programs suggest that the depths of sedations in training programs are "lightening." This target is laudable, but raises questions about efficacy for preschoolers for anything other than ultra-short procedures. Such use suggests a trend toward vastly increased use of general anesthesia, simply to avoid even the most remote possibility of an adverse or over-sedation result.

Agitation and need for frequent application of physical restraint to combat interfering movement from under-dosage or drug inadequacy as reported in these studies, or expectations that this agent will provide sufficient working times poses significant ethical concerns. At the least, need to resort to aversive measures to complete treatment under these conditions would seem to abandon the fundamental intent of sedation. Further conclusions declaring sedation success despite need for these interventions seem illogical.

Limitation of sedation regimen selection to a single or limited arsenal of available agents and the use of non-therapeutic dosing raises question about ethical and appropriate future use of sedation for the pediatric patient. Pro-active involvement of disciplines directly involved in the dental health care of the pediatric patient will ultimately determine the direction taken for continued use of sedation, either forwards or backwards. Continued assessment of the standards, proficiency, and experiences provided in advanced training programs seems warranted.

From the perspective of enforcement of compliance with established safety guidelines, efforts might be considered to establish national data banks requiring recording of mishaps and appraisals of etiology.

Lastly, imposition of more severe licensure sanctions might contribute incentive to lessening actions (described below) where guidelines are not followed.

\section{Case histories involving morbidity and mortality}

Case histories reported below are collected over the past fifteen years from published case reports, and court records. This is by no means a complete listing of untoward reactions and outcomes. It can be assumed that these represent a limited sample of occurrences nationwide. In each case, significant departures from standards of care and compliance with safety guidelines are evident. 
Citation: Nathan JE. The Direction of Pediatric Sedation: A Contemporary and Historical Look at its Science, Art, Strengths and Shortcomings. Inter J Otorhinolaryngology. 2015;2(1): 8.

Poor judgment with respect to agent selection and dosing and excess use of local anesthetic are found. With no exception, adverse reactions cited below are not explained by idiosyncratic drug reaction.

Case 1: 16 years old healthy but apprehensive female, weighing $60 \mathrm{~kg}$, presented for removal of impacted third molars. The patient received $3000 \mathrm{mg} \mathrm{CH}$ by mouth an hour before the procedure; IV pentothal was administered at the onset, and with administration

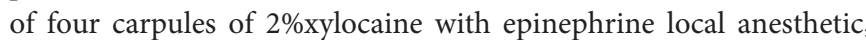
the patient manifested signs of convulsion, respiratory depression, followed by cardiac collapse and was pronounced dead two hours later. Preliminary depositions prior to trial revealed a lack of awareness of the clinician that $\mathrm{CH}$ was contraindicated indicated for children older than 6 years or weighing more than 60 pounds, that 50 $\mathrm{mg} / \mathrm{kg}$ dosing far exceeded the recommended maximal single dose. Combination with barbiturate, and local anesthesia were considered causative of the outcome.

Case 2: 3years old $22 \mathrm{~kg}$ female received 2,200 mg of CH (100 mg/ $\mathrm{kg}$ ) and $50 \%$ nitrous oxide, followed by 6 carpules of $2 \%$ Xylocaine with 1:100,000 epi. Under rubber dam, emesis was followed by aspiration, later convulsions, respiratory and cardiac arrest. No patient monitoring or sedation log was maintained no oxygen or emergency equipment available. Depositions suggested complete failure in compliance with sedation guidelines for agent and dosage selection, toxic dosage of local anesthetic exceeded, and no preparedness to recognize of manage a developing emergency.

Case 3: 14years old weight not recorded, received $1000 \mathrm{mg} \mathrm{CH}$ at home which prescribing dentist denied having prescribed (paper trail from pharmacy proved otherwise); $1000 \mathrm{mg} \mathrm{CH}$ administered on arrival when agitation behavior occurred; pt was deeply asleep when brought to operatory; no pt monitoring or sedation log; failure to recognize depth of sedation, respiratory depression, alteration of vital signs, and loss of protective reflexes; outcome: fatality.

Case 4: 4 years old weight unknown, male received $1000 \mathrm{mg}$ $\mathrm{CH}, 50 \mathrm{mg}$ Hydroxyzine, $50 \mathrm{mg}$ meperidine according to provider; no pharmacy record of agents prescribed; incomplete dental chart; no patient monitoring; no sedation log; no emergency kit available. Depositions revealed the pediatric dentist routinely stockpiled meds from other patients care for subsequent sedations; the dentist claimed that the "emergency kit was in the trunk of his car, parked across the street, and that that was considered within the standard of care." Assays revealed actual dosages were in range of $1500 \mathrm{mg} \mathrm{CH}$ and $100 \mathrm{mg}$ meperidine. Failure to recognize or manage respiratory depression and collapse, followed by cardiac arrest. Outcome: fatality. Availability and timely administration of the reversal agent may have been prevented the outcome.

Case 5: 5years old $35 \mathrm{lb}$ female received $7.5 \mathrm{mg}$ diazepam p.o. This was followed by IV placement and administration of $1.3 \mathrm{cc}$ or $1.3 \mathrm{mg}$ midazolam, $0.25 \mathrm{cc}$ atropine, and then $7.5 \mathrm{mg}$ pantazocine; this was followed by an additional IV dose of $6.5 \mathrm{mg}$ diazepam, followed by a second IV dose of $6.0 \mathrm{mg}$ diazepam. The dentist testified the pt was not awake, proceeded to perform the dental treatment, was transferred to recovery where the parent was brought in and realized her child was not breathing. Paramedics were called; the child was intubated, and taken to the hospital, later to be transferred to the Children's hospital where she was continued on a respirator till pronounced dead days later from anoxic encephalopathy. Minimal monitoring was reported, sedation record incomplete, and inadequate measures were taken to recognize and manage a developing emergency.

Case 6: 4years old $17.3 \mathrm{~kg}$ male received $1000 \mathrm{mg} \mathrm{CH}, 25 \mathrm{mg}$ hydroxyzine, $50 \mathrm{mg}$, meperidine followed by $50 \%$ nitrous oxide and four cartridges of $2 \%$ xylocaine with 1:100,000 epi. Respiratory depression was encountered, followed by convulsions and respiratory and cardiovascular collapse. The pt was revived and transported to an acute emergency care facility. Respirations were said to be spontaneous with signs of neurological abnormalities. The pt was later given a general anesthetic to permit intubation and airway stabilization to permit transfer to another facility. The pt was pronounced brain dead, and was later taken off the respirator. Combination of $1.97 \mathrm{X}$ toxic dosage of local anesthetic in combination with the sedative regimen was believed to be causative factors in the crisis.

The above cases demonstrate common factors which include serious drug interactions when narcotics are used for sedation, the importance of using appropriate concentrations of local anesthesia (without exceeding toxic doses for either anesthetic or included vasoconstrictor), attention to detail with respect to patient monitoring, responsible agent and dosage selection, proficiency and capacity to recognize and manage a complication should one arise.

Concerns arising from mishaps have gained increasing attention amongst health care providers within both dentistry and medicine. A listing of but a few of the many reports providing a critical look at sedation in dentistry is included in the references below for the interested reader [55-59].

It is noteworthy that a national watchdog group, the Raven Marie Blanco Foundation has brought attention in recent years to complications and deaths from sedation dentistry among children. Named for an 8 years old Virginia girl who died 90 minutes following a sedation procedure in 2007 for a tooth cleaning. It has compiled a list of 20 children that it claims have died since 1996. Among the problems remaining today is the tracking of deaths and complications from ill-conceived sedation practices. Legislation is lacking to establish national, state, or professional society databanks for mishaps, largely due to resulting litigation that restricts disclosure of events, outcomes, and settlements.

Analysis of sedation mishaps, as in the case reports included above and the existing literature, appears to carry a central theme: Poor if not deplorable and irresponsible clinician judgment, excessive dosage, inappropriate combinations of multiple agents, excessive use of local anesthetic, absence of appropriate patient monitoring and record keeping, lack of office preparedness and competency in recognition and management of medical crisis. Compliance failure to observe and implement existing safety guidelines appears rampant. Regimens and dosing used appear to have no ground in either science, or what is conceivably taught in advanced training programs. Of consequence, which has become problematical, is concomitant reaction of state regulatory bodies and institutions to further restrict the use of specific agents. Agents, which have over years of use demonstrated productive and safe outcomes, have been removed from the limited arsenal of agents currently available. 
Citation: Nathan JE. The Direction of Pediatric Sedation: A Contemporary and Historical Look at its Science, Art, Strengths and Shortcomings. Inter J Otorhinolaryngology. 2015;2(1): 8.

\section{Summation and Implications for Future Study}

The role and direction of pediatric dental conscious sedation appears to be reaching a crossroad.

While science and innovative research often clears the pathway and roadblocks to progress, it appears numerous obstacles remain. For every occurrence in which optimal or adequate results are obtained, helping some children to cope with invasive dental treatment, there appears to be others whereby sedation proves short of adequate necessitating use of aversive measures to complete treatment. Successful use of sedation has evolved into as much if not more so an art form than strictly science. For those gifted and skillful clinicians, selection of agents and dosages to produce effective and safe results, there seems less concern. For those lacking in background, experience and history of success predicting optimal agents and dosing schedules, it should not be surprising that these clinicians are more accepting of a need for the use of physical restraints and/ or more liberal selection of general anesthesia.

Disruptive behaviors, particularly from those lacking cooperative ability often are prompted by the need to protest and unpleasant situation and the impulse to protect oneself from perceived danger. The use of pharmacologic adjuncts to help avoid unpleasant and unproductive confrontations from the outset and to create an environment to facilitate the child's ability to ultimately accept care, protect the child's self esteem, foster a positive attitude toward care, and enhance the work quality of dental personnel can be frequently achieved with well selected sedation techniques.

Further controlled study of oral sedation regimens in current use which reflect improvement in research design methodology is needed. Attention to defining valid patient selection criteria with focus on variables pertinent to evaluating drug efficacy and safety can be expected to enhance clinician's choice of medication and dosing. A general consensus need be established in order to define what constitutes clinical success from beginning until fulfillment of discharge criteria.

There appears to be no general agreement among contemporary training programs with regard to agents or dosages universally considered effective for children simply on the basis of $\mathrm{mg} / \mathrm{kg}$ to guide clinician judgment. A controlled comparison between varying dosages for agents establishing low-, mid- and upper-range dosage limits is needed. Future determination made on the basis of the demands of a given visit for patients' apprehension level, issues currently unexplored, are needed to advance our knowledge and safe use of sedation for the pediatric patient. Variable experiences with sedation in training programs today may impact competency and safety outcomes in the area of pharmacologic management of pediatric patients. Strategies need be developed to strengthen consistency of competencies across academic training centers. In the private sector, mechanisms to insure and verify evidence of provider and facility proficiency in emergency management on a valid and timely basis, while bureaucratic and time consuming, is warranted. Current limited if not almost exclusive use of benzodiazepines using the oral route is in need of re-assessment. Expansion to include testing of a diverse arsenal of agents seems warranted for procedures of short duration as well as longer duration. The latter seems particularly urgent in view of state and institutional restriction of the use of a widely studied and abused agent.

\section{References}

1. Nathan JE (1995) Managing behavior of pre-cooperative children. Dent Clin North Am 39: 789-816.

2. Weinstein P, Nathan JE (1988) The challenge of fearful and phobic children Dent Clin North Am 32: 657-692.

3. Nathan JE (2001) Behavioral management strategies of young pediatric dental patients with disabilities. ASDC J Dent Child 68: 89-101.

4. Nathan JE (2003) Pediatric Dental Sedation: when non-pharmacologic approaches prove inadequate or inappropriate, guest editorial. J Pediatric Dent Care 9: 8-9.

5. Nathan JE (1991) Oral conscious sedation for the pediatric dental patient Update Pediatr Dent 4: 1-4, 6-8.

6. American Academy of Pediatric Dentistry, Educational Foundation (1988) Behavior management for the pediatric dental patient, current issues and implications for the future - a conference workshop. Iowa City, IA

7. Nathan JE (1999) Dosage selection for Pediatric Oral Conscious sedation: A practical approach. J Southeastern Soc Pediatr Dent 5: 27.

8. Wilson S, Nathan JE (2011) A survey study of sedation training in advanced pediatric dentistry programs: thoughts of program directors and students. Pediatr Dent 33: 353-360.

9. Nathan JE, West MS (1987) Comparison of Chloral hydrate-hydroxyzine with and without meperidine for management of the difficult pediatric dental patient. ASDC J Dent Child 54: 437-444.

10. Needleman HL Joshi A, Griffith DG (1995) Conscious sedation of pediatric dental patients using chloral hydrate, hydroxyzine and nitrous oxide - a retrospective study of 382 sedations. Pediatr Dent 17: 424-431.

11. Wilson S (2004) Pharmacologic management of the pediatric dental patient Pediatr Dent 26: 131-136.

12. Wright GZ, McAulay D (1973) Current premedicating trends in pedodontics ASDC J Dent Child 40: 185-187.

13. Association of Pedodontic Diplomates (1981) Survey of techniques for behavior management. Pediatr Dent 3: 246.

14. Duncan WK, Pruhs RJ, Ashrafi MH, Post AC (1983) Chloral hydrate and other drugs used in sedating young children: a survey of Diplomates. Pediatr Dent 5: 252-256.

15. Davis MJ (1988) Conscious sedation practices in pediatric dentistry : a survey of the members of the American Board of Pediatric Dentistry College of Diplomates. Pediatr Dent 10: 328.

16. Nathan JE (1989) Management of the difficult child: A survey of pediatric dentists' use of restraints, sedation, and general anesthesia. ASDC J Dent Child 56: 293-301.

17. Wilson S, McTigue DJ (1989) Survey of conscious sedation practices in pediatric dentistry advanced residency programs. J Dent Educ 53:595-597.

18. Acs G, Musson CA, Burke MJ (1990) Current teaching of restraint and sedation in pediatric dentistry: a survey of program directors. Pediatr Dent 12: $364-367$

19. Wilson S (1996) A survey of the American Academy of Pediatric Dentistry membership: Nitrous oxide and sedation Pediatr Dent 18:287-293.

20. Carr KR, Wilson S, Nimer S, Thornton JB Jr (1999) Behavior management techniques among pediatric dentists practicing in the southeastern U.S. Pediatr Dent 21:347-353.

21. Wilson S, Farrell K, Griffen A, Coury D (2001) Conscious sedation experiences in graduate pediatric dentistry programs. Pediatr Dent 23: 307-314.

22. Houpt M (2002) Project USAP 2000 --Use of sedative agents by pediatric dentists: a 15 year follow-up survey. Pediatr Dent 24: 289-294. 
Citation: Nathan JE. The Direction of Pediatric Sedation: A Contemporary and Historical Look at its Science, Art, Strengths and Shortcomings. Inter J Otorhinolaryngology. 2015;2(1): 8.

ISSN: 2380-0569

23. Adair SM, Rockman RA, Schafer TE, Waller JL (2004) Survey of behavior management techniques in pediatric dentistry advanced education programs. Pediatr Dent 26: 151-158.

24. Rhodes A, Wilson S (2004) Research in pediatric dental postgraduate programs and residencies: results of a survey. Pediatr Dent 26: 75-78

25. Tobias M, Lipschultz DH, Album MM (1975) A Study of three pre-operative sedation combinations. J Dent Child 42: 453-459.

26. Trapp LD (1982) Pharmacologic management of pain and anxiety. In Stewart Troutman, Barber and Wei, Pediatric Dentistry: Scientific Foundations and Clinical Practice. Philadelphia: WB Saunders, p: 910-832.

27. Musselman RJ, McClure DB (1975) Pharmaco-therapeutic approaches to behavior management of the pediatric dental patient. In Wright, GZ, ed, Behavior Management in Dentistry for Children. Philadelphia: Saunders, $p$ : 147

28. Musselman RJ (1991) Considerations in behavior management of the pediatric dental patient: helping children cope with dental treatment. Pediat Clin North Am 38: 1309-1324.

29. Goodson JM, Moore PA (1983) Life-Threatening Reactions after pedodontic sedation: an assessment of narcotic, local anesthetic, and anti-emetic drug interaction. J Am Dent Assoc 107: 239-245.

30. Moore PA (1984) Comparisons of sedation regimens for the pediatric patient An assessment tool. J Am Dent Assoc 109: 564-569.

31. Houpt MI, Sheskin RB, Koenigsberg SR, Desjardins PJ, Shey Z (1985) Assessing chloral hydrate dosage for young children. ASDC J Dent Child 52 364-369.

32. Moody EH, Mourino AP, Campbell R (1986) The therapeutic effectiveness of nitrous oxide and chloral hydrate administered orally, rectally and combined with hydroxyzine for pediatric dentistry. ASDC J Dent Child 53: 425-429.

33. Badalaty MM, Houpt MI, Koenigsberg SR, Maxwell KC, DesJardins PJ (1990) Comparison of chloral hydrate dosage for young children. Pediatr Dent 12 : 33-37.

34. Meyer ML, Mourino AP, Farrington FH (1990) Comparison of triazolam to chloral hydrate/hydroxyzine combination in the sedation of pediatric dental patients. Pediatr Dent 12: 283-287.

35. Meyer ML, Mourino AP, Farrington FH (1990) Comparison of triazolam to chloral hydrate, hydroxyzine combinations in the sedation of pediatric dental patients. Pediatr Dent 12: 283-287.

36. Poorman T, Farrington FH, Mourino AP (1990) Comparison of chlora hydrate-hydroxyzine combination with and without meperidine in the sedation of pediatric dental patients. Pediatr Dent 12: 288-291.

37. Robbins (1967) Comparison of chloral hydrate with and without promethazine J Dent Child 34:150.

38. Hasty MF, Vann WF Jr, Dilley DC, et al. (1990) Evaluation of two drug regimens to sedate pediatric dental patients. Pediatr Dent 12: 342

39. Hasty MF, Vann WF Jr, Dilley DC, Anderson JA (1991) Conscious sedation of pediatric dental patients: An investigation of Chloral hydrate-hydroxyzine pamoate and meperidine vs chloral hydrate and hydroxyzine pamoate. Pediatr Dent 13: 10-19.

40. Tsinidou KG, Curzon ME, Sapsford DJ (1992) A study to compare the effectiveness of temazepam and a chloral hydrate combination in sedating pediatric dental patients. Int J Paediatr Dent 2: 163-169.

41. Wilson S (1992) Chloral hydrate and its effects on multiple physiologic parameters in children: A dose-response study. Pediatr Dent 14: 171-177.
42. Gladney M, Stanley RT, Hendricks SE (1994) Anxiolytic activity of chlora hydrate and hydroxyzine. Pediatr Dent 16: 183-189.

43. Wilson S, Easton J, Lamb K, Orchardson R, Casamassimo P (2000) A retrospective study of chloral hydrate, hydroxyzine, and meperidine, and midazolam regimens used to sedate children for dental care. Pediatr Dent 22: 107-112.

44. Cathers JW, Wilson CF, Webb MD, Alvarez ME, Schiffman T, et al. (2005) A comparison of two meperidine/hydroxyzine sedation regimens for the uncooperative pediatric dental patient. Pediatr Dent 27: 395-400.

45. Sheroan MM, Dilley DC, Lucas WJ, Vann WF (2006) A prospective study of 2 sedation regimens in children: chloral hydrate, meperidine, and hydroxyzine vs midazolam, meperidine and hydroxyzine. Anesth Prog 53: 83-90.

46. McCormack L, Chen JW, Trapp L, Job A (2014) A Comparison of Sedation related events for two Multi-agent oral sedation regimens in pediatric dental patients. Pediatr Dent 36: 302-308.

47. Silver T, Wilson C, Webb M (1994) Evaluation of two doses of midazolam as a conscious sedation for physically and neurologically compromised pediatric dental patients. Pediatr Dent 16: 350- 359

48. Rai K, Hegde AM, Goel K (2007) Sedation in uncooperative children undergoing dental procedures: A comparative evaluation of midazolam, propofol, and ketamine. J Clin Pediatr Dent 32: 1- 4.

49. Wilson KE, Welbury RR, Girdler NM (2002) A randomized controlled trial of oral midazolam and nitrous oxide for Pediatric dental sedation. Anaesthesia 57: 860-867

50. Somri M, Parisinos CA, Kharouba J, Cherni N, Smidt A, et al. (2012) Optimizing the dose of oral midazolam sedation for dental procedures in Children: a prospective, randomized controlled study. Int J Paediatr Dent 22 271-279.

51. Peretz B, Kharouba J, Somri M (2014) A comparison of two different dosages of midazolam. Pediatr Dent 36: 228-232.

52. Nathan JE (2003) Issues affecting agent and dosage selection for pediatric conscious sedation. J Southeastern Soc Pediatr Dent 9: 17-19

53. Nathan JE (1999) Pediatric Oral Conscious Sedation: What works and what's safe. J Southeastern Soc Pediatr Dent 5: 26-27.

54. Casamassimo PS (2012) Guest editorial: The policy center story - My version. Pediatr Dent 34: 10-11.

55. Cote CJ (1994) Sedation for the pediatric patient: a review. Pediatr Clin North Am 41: 31-58.

56. Pershad J, Palmisano P, Nichols M (1999) Chloral hydrate: The good and the bad. Pediatr Emerg Care 15: 432-435.

57. Coté CJ, Karl HW, Notterman DA, Weinberg JA, McCloskey C (2000) Adverse sedation events in pediatrics: analysis of medications used in sedation. Pediatrics 106: 633-644

58. Dionne RA, Yagiela JA, Coté CJ, Donaldson M, Edwards M, et al. (2006) Balancing efficacy and safety in the use of oral sedation in dental out-patients. J Am Dent Assoc 137: 502-513.

59. Cravero JP, Blike GT, Beach M, Gallagher SM, Hertzog JH, et al. (2006) Incidence and nature of adverse events during pediatric sedation/anesthesia for procedures outside the operating room, report from the Pediatric Sedation Research Consortium. Pediatrics 118: 1087-1096.

60. Nathan JE, Vargas KG (2002) Oral Midazolam with and without meperidine for management of difficult young pediatric dental patients: a retrospective study. Pediatr Dent 24: 129-138. 